\title{
A numerical scheme for moving boundary problems that are gradient flows for the area functional
}

\author{
UWE F. MAYER \\ Department of Mathematics, Vanderbilt University, Nashville, TN 37240, U.S.A.
}

(Received Received 24 April 1997; revised 12 February 1999)

\begin{abstract}
Many moving boundary problems that are driven in some way by the curvature of the free boundary are gradient flows for the area of the moving interface. Examples are the Mullins-Sekerka flow, the Hele-Shaw flow, flow by mean curvature, and flow by averaged mean curvature. The gradient flow structure suggests an implicit finite differences approach to compute numerical solutions. The proposed numerical scheme will allow to treat such free boundary problems in both $\mathbb{R}^{2}$ and $\mathbb{R}^{3}$. The advantage of such an approach is the re-usability of much of the setup for all of the different problems.

As an example of the method we will compute solutions to the averaged mean curvature flow that exhibit the formation of a singularity.
\end{abstract}

\section{Introduction}

In this paper we will study geometric evolution problems for surfaces driven by curvature. These moving boundary problems arise from models in physics and the material sciences, and they describe phase changes, or more generally, phenomena in fluid flow.

The Hele-Shaw model (named after H.S. Hele-Shaw [47]) describes the pressure of two immiscible viscous fluids trapped between two parallel glass plates and has attracted considerable attention in the literature, both on the analytical side [23, 27, 28, 29, 33, $38,51,54,63]$ and on the numerical side $[2,6,7,8,12,25,26,49,50,65]$. This list of references also encompasses work related to the one-sided Hele-Shaw model, which arises as a limit when the viscosity of one of the fluids approaches zero. Furthermore, the Hele-Shaw model has classically been considered on a bounded domain, but many of the references above also address the problem on an unbounded domain. We shall only consider the problem on all of $\mathbb{R}^{n}$; for the precise formulation see (4.4).

The Mullins-Sekerka model was first proposed by (and much later named after) Mullins and Sekerka to study solidification of materials of negligible specific heat [58]. For a while this model was also called the Hele-Shaw model, leading to a somewhat confused literature. Pego [61], and then Alikakos, Bates, and Chen [3], and also Stoth [68], established this model as a singular limit of the Cahn-Hilliard equation [17], a fourth order partial differential equation modelling nucleation and coarsening phenomena in a melted binary alloy. The Mullins-Sekerka model is considered to be a good model to describe the stage of Ostwald ripening in phase transitions, which is the stage after the initial nucleation has 
essentially been completed and where some particles grow at the cost of others in an effort to decrease interfacial energy (surface area). The literature focuses mainly on the modelling and analytical aspects of the problem $[11,16,18,19,21,32,34,46,56,66,71,72]$ though there are numerical simulations for the two-dimensional case [10, 73]. We shall consider the two-phase problem on all of $\mathbb{R}^{n}$, see (4.3).

The mean curvature flow is probably the most studied geometric moving boundary problem, it is in some way also the simplest: the normal velocity is equal to the mean curvature of the interface. This sharp interface model arises, for example, as a singular limit of the Allen-Cahn equation [4], see the paper by Evans, Soner, and Souganidis [36]. Physically, the Allen-Cahn equation describes the motion of phase-antiphase boundaries between two grains in a solid material. There is also a volume preserving version of the mean curvature flow, for which one subtracts the average of the mean curvature from the normal velocity. A plethora of analytical results and theories of weak solutions exists, for example, see [20, 22, 30, 35, 36, 37, 43, 44, 52, 53, 57, 67], and for numerical solutions $[1,9,13,14,15,60,69]$, to name but a few.

The algorithm proposed below is a front-tracking boundary-integral method. It has the advantage that one does not have to differentiate across the front, as compared to a level-set approach. Also, at least if the inner product (see below) is of local character, it makes no difference whether the moving interface is immersed or embedded. Both fronttracking methods and the level-set methods have been used intensively in the literature, see for example the recent review article by Hou [48].

Now let us describe what we mean under a gradient flow for the area of the free boundary. First of all, area means surface area if the problem setting is $\mathbb{R}^{3}$, or length if the setting is $\mathbb{R}^{2}$. The notion of gradient depends on a function space defined on the free boundary. This function space is assumed to be a (pre-)Hilbert space $\mathbf{H}$ contained in $\mathbf{L}_{2}$ of the free boundary $\Gamma(t)$. Each (sufficiently small) element of $\mathbf{H}$ gives rise to a graph in the normal direction over $\Gamma(t)$, and one can compute the area of that graph. Under suitable assumptions this functional, which maps functions on the interface to the area of the resulting graph, is differentiable, and its derivative, being linear, can be represented by a vector in the space $\mathbf{H}$. This gradient vector clearly depends on the metric of $\mathbf{H}$ and is of course an element of $\mathbf{H}$, that is a function on $\Gamma(t)$. If now for all times $t$ the normal velocity of the moving boundary coincides with the negative gradient of the area functional with respect to $\mathbf{H}(\Gamma(t))$, then this free boundary problem is considered a gradient flow for the area. This notion is due to P. Fife [39, 40]. The free boundary problems mentioned above fall within the characterisation as gradient flows.

We propose to replace the normal velocity by a finite difference, and the scheme should be chosen to be implicit. That means that the computation of the velocity will depend on the interface at the next time step. This is essential for the stability of the computations. This dependence will then be replaced by an approximate linearised dependence, which makes the system semi-implicit. Finally, discretisation of the surface in the spatial variables and then averaging over (small) patches of the surface will lead to a linear system for the velocities of the surface patches. This is where the nature of the operator $S$ giving the scalar product of $\mathbf{H}$ plays an essential role. We will assume that either $S$ is of local character, or that one of $S$ or $S^{-1}$ has an integral representation on the interface. For the mean curvature flow $S$ is local; for the Mullins-Sekerka flow $S$ has an integral represen- 
tation with a kernel that happens to be independent of the free boundary, while for the Hele-Shaw problem there is an integral representation for $S^{-1}$ with a kernel depending only in a local manner on the unknown interface. The special form of the kernels is of practical importance for the spatial discretisation.

Some of these ideas have been implicitly used by Bates, Chen, and Deng [10] for the two-sided Mullins-Sekerka problem in two space dimensions (the interface is a curve in the plane and spatial discretisation amounts to approximating a curve by a polygon). However, their approach is ad-hoc and does not address the gradient flow nature of the problem. Also for the Mullins-Sekerka in two space dimensions, there is the refined method by Zhu, Chen, and Hou [73] which avoids the numerically laborious step of inversion of a matrix to compute the next time step. The idea of a semi-implicit scheme appears also elsewhere in the literature, see for example $[8,6,50]$. Of particular interest is a paper by Petersson [62], in which he computes numerical solutions to a free boundary problem in the plane. There he uses the idea of adding a perturbation of order $\epsilon$ to a solution and expanding the perturbed solution in $\epsilon$. Collecting first order terms in $\epsilon$ leads then to a semi-implicit scheme for the perturbation. This is clearly similar in spirit to what we do below. We consider the interface at the next time step as a normal variation of the current interface, and then compute a linear approximation of the curvature of the interface at the next time step. However, we have more structure in our equations, and it turns out we need to linearise only the curvature, and no other state variable. This then leads to a simple and elegant iterative scheme because we have a linear relationship between curvature and normal velocity. This is, in fact, the main advantage of the gradient flow structure, because all nonlocal and nonlinear dependencies can be hidden away in the inner product.

Of related interest is the paper by Almgren, Taylor, and Wang [5], in which another variational approach to curvature driven flows is given. Finally, for an overview of other methods used to treat geometric moving interface problems the reader is referred to the paper by Taylor, Cahn, and Handwerker [70].

\section{Analytical background material}

The author owes much of the material in this section to P. Fife $[39,40]$.

\subsection{Definition of Gradient Flows}

Gradient flows are a natural model for the dynamics of a system that is known to cause some quantity $\mathcal{A}$ to decrease as time evolves; that is, one seeks to solve

$$
\frac{d u(t)}{d t}=-\nabla \mathcal{A}(u(t))
$$

where $u(t)$ describes the state of the system. In this paper the system will be characterised by a compact $(n-1)$-dimensional surface $\Gamma(t)$ embedded or immersed in $\mathbb{R}^{n}$, which need not necessarily be connected, and $\mathcal{A}$ will be the $(n-1)$-dimensional surface area functional. We are interested in the physically relevant cases $n=2$ or $n=3$. We assume 
the evolution is sufficiently smooth, that is, for small enough times $\tau$ we have

$$
\Gamma(t+\tau)=\left\{x \in \mathbb{R}^{n}: x=y+\rho(\tau, y) N(t, y), y \in \Gamma(t)\right\}
$$

for some smooth function $\rho$ and unit outer normal $N$ of $\Gamma(t)$. Let $V(t, y)=\left.\frac{\partial \rho}{\partial \tau}(\tau, y)\right|_{\tau=0}$ denote the normal velocity of $\Gamma(t)$. The following two basic formulae from differential geometry are the starting point:

$$
\begin{aligned}
\frac{d}{d t} \mathcal{A}(\Gamma(t)) & =\int_{\Gamma(t)} V(x) \kappa(x) d \sigma_{x}, \\
\frac{d}{d t} \operatorname{Vol}(\Gamma(t)) & =\int_{\Gamma(t)} V(x) d \sigma_{x} .
\end{aligned}
$$

Here $\kappa$ denotes the curvature of $\Gamma(t)$ if $n=2$, and the mean curvature of $\Gamma$ if $n=$ 3. Throughout this paper the mean curvature is defined as the sum of the principal curvatures; this avoids having a factor of $(n-1)$ in formula $(2.1)$. The sign is chosen so that a sphere has positive curvature with respect to the outer normal; by the definition of $V$ a family of expanding spheres has positive normal velocity. $\operatorname{Vol}(\Gamma(t))$ denotes the $n$-dimensional volume enclosed by $\Gamma(t)$.

For any fixed $\Gamma$ consider a (pre-)Hilbert space $\mathbf{H}(\Gamma) \subset \mathbf{L}_{2}(\Gamma)$. The right-hand side of equation (2.1) can be interpreted as a linear functional for the function $V$,

$$
d \mathcal{A} / d t: \mathbf{H}(\Gamma) \rightarrow \mathbb{R}, \quad V \mapsto \int_{\Gamma} V \kappa d \sigma
$$

In some cases this will be a bounded linear functional on $\mathbf{H}(\Gamma)$. We assume that this is the case and that we can represent the functional as the scalar product with some unique element $\nabla_{\mathbf{H}(\Gamma)} \mathcal{A}$. By the Riesz representation theorem this is certainly possible if $\mathbf{H}(\Gamma)$ is complete. Hence for any $v \in \mathbf{H}(\Gamma)$

$$
\int_{\Gamma} v \kappa d \sigma=\left\langle v, \nabla_{\mathbf{H}(\Gamma)} \mathcal{A}>_{\mathbf{H}(\Gamma)},\right.
$$

and in particular the following equation holds:

$$
\frac{d \mathcal{A}}{d t}=\left\langle V, \nabla_{\mathbf{H}(\Gamma)} \mathcal{A}>_{\mathbf{H}(\Gamma)} .\right.
$$

$\Gamma(t)$ is said to be a gradient flow for $\mathcal{A}$ if for all times $t$

$$
V(t)=-\nabla_{\mathbf{H}(\Gamma(t))}(\mathcal{A})
$$

Notice that the space with respect to which the gradient is computed is constantly changing.

As the easiest example set $\mathbf{H}(\Gamma(t))=\mathbf{L}_{2}(\Gamma(t))$. From equation (2.3) we have $\langle v, \kappa\rangle=$ $<v, \nabla_{\mathbf{H}(\Gamma(t))} \mathcal{A}>$ with both inner products in $\mathbf{L}_{2}(\Gamma(t))$, therefore $\nabla_{\mathbf{H}(\Gamma(t))} \mathcal{A}=\kappa$ holds, and the $\mathbf{L}_{2}$-gradient flow is given by $V=-\kappa$. This is the usual flow by mean curvature. 


\subsection{Volume preserving flows}

We write a superscript zero when we talk about a function space containing only functions with average zero. Zero-average subspaces are particularly important because gradient flows in these spaces preserve the enclosed volume by (2.2).

Let $S$ be a positive definite symmetric operator whose domain and range are (dense) linear subspaces in $\mathbf{L}_{2}^{0}(\Gamma)$. Define $\mathbf{H}(\Gamma)$ to be the (pre-)Hilbert space consisting of the functions in the domain of $S$ endowed with the scalar product $\left\langle u, w>_{\mathbf{H}(\Gamma)}=\langle u, S w\rangle_{\mathbf{L}_{2}^{0}(\Gamma)}\right.$. Denote the average of $\kappa$ on $\Gamma$ by $\bar{\kappa}$. Then for $v \in \mathbf{H}(\Gamma)$, using (2.3) for the first equality,

$$
\left\langle v, \nabla_{\mathbf{H}(\Gamma)} \mathcal{A}>_{\mathbf{H}(\Gamma)}=\left\langle v, \kappa>_{\mathbf{L}_{2}}=\left\langle v, \kappa-\bar{\kappa}>_{\mathbf{L}_{2}^{0}}=\left\langle v, S^{-1}(\kappa-\bar{\kappa})>_{\mathbf{H}(\Gamma)},\right.\right.\right.\right.
$$

and the gradient flow with respect to the space $\mathbf{H}$ is given by

$$
V=-S^{-1}(\kappa-\bar{\kappa}) \text {. }
$$

\section{Discretisation}

The proposed scheme for the gradient flows involves essentially two steps. First discretise in time, and then triangulate the surface (if $n=3$, for $n=2$ approximate the curve by a polygon). In this outline we will only treat volume preserving flows that can be set up as outlined in Section 2.2. The notation of Section 2 will be used below.

\subsection{Finite differences}

The main idea for discretisation in time is as follows. For some abstract gradient flow

$$
\frac{d u(t)}{d t}=-\nabla \mathcal{A}(u(t))
$$

one replaces the time derivative by a finite difference

$$
\frac{u(t+h)-u(t)}{h}=-\nabla \mathcal{A}(u(t+h)) .
$$

Hence one has formally

$$
u(t+h)=(\mathrm{id}+h \nabla \mathcal{A})^{-1} u(t)
$$

here id stands for the identity map. Now set $h=t / k$, then it can be expected that

$$
u(t) \stackrel{\text { def }}{=}\left(I+\frac{t}{k} \nabla \mathcal{A}\right)^{-k} u(0)
$$

is a good approximation for the exact solution $u_{\text {exact }}(t)$ at time $t$. One can show that under suitable assumptions the exact solution is given by the exponential formula

$$
u_{\text {exact }}(t)=\lim _{k \rightarrow \infty}\left(I+\frac{t}{k} \nabla \mathcal{A}\right)^{-k} u(0),
$$

see for example $[24,55]$. This motivates our numerical approach here, even as the setting in those two references is essentially different.

First we introduce a bit of notation. Let $\Sigma$ be a fixed manifold immersed or embedded in $\mathbb{R}^{n}$ and assume $\Gamma(t)$ is a parameterised as a graph in the normal direction over $\Sigma$, that 
is $\Gamma(t)=\operatorname{im}(\phi(., t))$ with $\phi: \Sigma \times(t-\epsilon, t+\epsilon) \rightarrow \mathbb{R}^{n}$. In fact, for the computations we will simply use $\Sigma=\Gamma(t)$, but for the derivation is is better to keep the roles separate. For free boundary problems the time derivative of an abstract evolution problem corresponds to the normal velocity, hence equation (2.5) yields the following finite difference equation

$$
N(\phi(x, t), t) \cdot \frac{\phi(x, t+h)-\phi(x, t)}{h}=-S^{-1}(\kappa(., t+h)-\bar{\kappa}(t+h))(\phi(x, t+h)) .
$$

One should actually write $\widetilde{\phi}$ and so on because the above equation describes approximate solutions; by a similar abuse of notation we will call the left side of the above equation $V(\phi(x, t), t)$ again and hence

$$
\begin{aligned}
\phi(x, t+h) & =\phi(x, t)+h V(\phi(x, t), t) N(\phi(x, t), t), \\
V(\phi(x, t), t) & =-S^{-1}(\kappa(., t+h)-\bar{\kappa}(t+h))(\phi(x, t+h)) .
\end{aligned}
$$

Finally approximate the dependency of the curvature of the next time step by

$$
\kappa(\phi(x, t+h), t+h) \approx \kappa(\phi(x, t), t)+h L V(\phi(x, t))
$$

where $L$ is a linear operator which is formally defined via

$$
L(V(\phi(x, t), t))=\left.\frac{d}{d h} \kappa(\phi(x, t)+h V(\phi(x, t), t) N(\phi(x, t), t))\right|_{h=0} .
$$

As $V(., t)$ is in the range of $S^{-1}$ it has average zero, and hence by linearity of $L$ so does $L(V(., t))$, thus

$$
V(., t)=-S^{-1}(\kappa(., t)+h L(V(., t))-\bar{\kappa}(t)) .
$$

This finally leads to the (semi-)implicit scheme given by (here id stands for the identity map)

or equivalently

$$
\left(\mathrm{id}+h S^{-1} L\right)(V(., t))=-S^{-1}(\kappa(., t)+\bar{\kappa}(t))
$$

$$
(S+h L)(V(., t))=-\kappa(., t)+\bar{\kappa}(t) .
$$

It should be mentioned here, that it is now clear that this setup not only works for gradient flows, but also for others, that are given by a not necessarily invertible linear operator $A$ defined on $\mathbf{L}_{2}$ or $\mathbf{L}_{2}^{0}$, such that

$$
V=-A(\kappa) \quad \text { or } \quad V=-A(\kappa-\bar{\kappa}) .
$$

The scheme is then the one given by (3.2) with $S^{-1}$ replaced by $A$,

$$
(I+h A L)(V(., t))=-A(\kappa(., t)+\bar{\kappa}(t)) .
$$

However, if $A$ is not linear, then this elegant setup no longer works. The point of this paper is that gradient flows provide an interesting class of free boundary problems satisfying (3.4).

\subsection{Spatial discretisation}

As mentioned above, let $\Sigma=\Gamma(t)$, so that in particular $\phi(x, t)=x$. Subdivide the interface $\Gamma$ into a finite collection of disjoint surface patches (line segments) $\Gamma_{i}$ and choose 
points $z_{i} \in \Gamma_{i}$. Let $\left|\Gamma_{i}\right|$ denote the surface area (length) of $\Gamma_{i}$. Use the approximations

$$
\begin{aligned}
\kappa\left(z_{i}, t\right) & \approx \frac{1}{\left|\Gamma_{i}\right|} \int_{\Gamma_{i}} \kappa(x, t) d \sigma_{x} \\
\bar{\kappa}(t) & \approx \sum_{i} \frac{\left|\Gamma_{i}\right|}{|\Gamma|} \kappa\left(z_{i}, t\right)
\end{aligned}
$$

and average equation (3.3). This leads to

$$
\frac{1}{\left|\Gamma_{i}\right|} \int_{\Gamma_{i}}(S+h L)(V(x, t)) d \sigma_{x}=-\kappa\left(z_{i}, t\right)+\bar{\kappa}(t) .
$$

Assume $S$ to be an integral operator, that is, there is a function $k(x, y)$ associated with $S$ so that for any suitable function $w(x)$ one has

$$
(S w)(x)=\int_{\Gamma} k(x, y) w(y) d \sigma_{y} .
$$

Approximate further by using $V(y, t) \approx V\left(z_{j}, t\right)$ for $y \in \Gamma_{j}$. This leads to

$$
\frac{1}{\left|\Gamma_{i}\right|} \int_{\Gamma_{i}} S V(x, t) d \sigma_{x} \approx \frac{1}{\left|\Gamma_{i}\right|} \sum_{j} V\left(z_{j}, t\right) \int_{\Gamma_{i}} \int_{\Gamma_{j}} k(x, y) d \sigma_{y} d \sigma_{x}
$$

Finally, as $L$ is a linear mapping on $V$ we seek an approximation of the form

$$
\sum_{j} l_{i j} V\left(z_{j}, t\right) \approx \frac{1}{\left|\Gamma_{i}\right|} \int_{\Gamma_{i}} L V(x, t) d \sigma_{x}
$$

for some coefficients $l_{i j}$ depending on $\Gamma(t)$ but independent of $V(x, t)$. If we also define

$$
s_{i j}=\frac{1}{\left|\Gamma_{i}\right|} \int_{\Gamma_{i}} \int_{\Gamma_{j}} k(x, y) d \sigma_{y} d \sigma_{x}
$$

then we obtain a linear system for the unknown velocity at the points $z_{i}$

$$
\forall i: \quad \sum_{j}\left(s_{i j}+h l_{i j}\right) V\left(z_{j}, t\right)=-\kappa\left(z_{i}, t\right)+\bar{\kappa}(t) .
$$

In case it is not $S$ but $S^{-1}$ which is an integral operator it will be preferable to start from (3.2) and to proceed as above. If we use $k(x, y)$ to denote the kernel corresponding to $S^{-1}$ and use equation (3.7) again to define the matrix now belonging to $S^{-1}$, then the numerical scheme is given by

$$
\forall i: \quad \sum_{j}\left(\delta_{i j}+\sum_{k} h s_{i k} l_{k j}\right) V\left(z_{j}, t\right)=-\sum_{j} s_{i j}\left(\kappa\left(z_{j}, t\right)-\bar{\kappa}(t)\right) .
$$

\subsection{Approximation of the curvature}

The surface will be represented as a list of vertices $\left\{z_{1}, \ldots, z_{m}\right\}$, edges, and, if $n=3$, oriented triangular facets. Furthermore, if $n=3$, then every edge is assumed to belong to exactly two facets; a vertex, however, can belong to arbitrarily many facets.

The (mean) curvature is considered to be located at the vertices. The motivation of the formula used is as follows. Let $\eta$ be a piecewise linear function of one real variable 
with $\eta(0)=1$ and $\eta(t)=0$ for $|t|>\epsilon$. Pick a point $z$ on a smooth surface $\Gamma$ embedded in $\mathbb{R}^{n}$ and consider the variation of $\Gamma$ with variation field $V(x)=\eta(|x-z|) N(x)$. Then by $(2.1)$

$$
\frac{d \mathcal{A}}{d t}=\int_{B_{\epsilon}(z)}\left(1-\frac{1}{\epsilon}|x-z|\right) \kappa(x) d \sigma_{x} \approx \omega_{n-1} \kappa(z) \int_{0}^{\epsilon}\left(1-\frac{\rho}{\epsilon}\right) \rho^{n-2} d \rho=\frac{\omega_{n-1}}{n^{2}-n} \pi \epsilon^{n-1} \kappa(z) .
$$

Here $\omega_{n-1}$ stands for the surface area of the unit sphere in $\mathbb{R}^{n}$. For the following assume $n=3$. On the triangulated surface the same effect of this variation can be achieved by the (normal) movement of one vertex. The star of a vertex $z$ is the union of all facets containing $z$, and we choose $\epsilon$ from above in such a way that the area of $\operatorname{star}(z)$ equals the area of the $\epsilon$-ball about $z$. Consider the area of the triangulated surface as a function of the position of $z$, all other vertices are fixed. It is clear that the biggest rate of change occurs under normal variations for a given magnitude of variation, hence

$$
\left|\frac{d \mathcal{A}}{d t}\right| \approx\left|\nabla_{z} \mathcal{A}\right|
$$

The reasoning for $n=2$ proceeds analogously, and the final formula for the mean curvature at the vertex $z_{i}$ then is (where $N_{i}$ denotes the outer normal at $z_{i}$ )

$$
\begin{array}{ll}
\kappa_{i}=\frac{\nabla_{z_{i}} A_{i} \cdot N_{i}}{\left(\left|z_{i}-z_{i-1}\right|+\left|z_{i}-z_{i+1}\right|\right) / 2} & \text { for } n=2, \\
\kappa_{i}=\frac{\nabla_{z_{i}} A_{i} \cdot N_{i}}{\left(\text { area of } \operatorname{star}\left(z_{i}\right)\right) / 3} & \text { for } n=3 .
\end{array}
$$

For the case $n=3$ this formula for the mean curvature can also be derived using the soap film model for the triangulated surface. There $\nabla_{z_{i}} \mathcal{A}$ is the force due to surface tension at the vertex $z_{i}$, and to get the mean curvature one divides by one-third of the area of the star as each facet belongs to three stars. This interpretation appears in [15].

Now for $n=3$ let $A_{i}=$ area of $\operatorname{star}\left(z_{i}\right)$, then

$$
A_{i}=\frac{1}{2} \sum\left|\left(z_{j}-z_{i}\right) \wedge\left(z_{k}-z_{i}\right)\right|
$$

where the sum ranges over those indices so that $z_{i}, z_{j}$, and $z_{k}$ span a positively oriented facet of $\operatorname{star}\left(z_{i}\right)$. The outward normals on the facets are given by

$$
N_{i j k}=\frac{\left(z_{j}-z_{i}\right) \wedge\left(z_{k}-z_{i}\right)}{\left|\left(z_{j}-z_{i}\right) \wedge\left(z_{k}-z_{i}\right)\right|}
$$

A standard computation leads to

$$
\nabla_{z_{i}} \mathcal{A}=\nabla_{z_{i}} A_{i}=\frac{1}{2} \sum\left(N_{i j k} \wedge\left(z_{k}-z_{j}\right)\right) .
$$

The outward pointing vector of $\pm \nabla_{z_{i}} A_{i} /\left|\nabla_{z_{i}} A_{i}\right|$ can then be defined to be the unit outer normal $N_{i}$. For $n=2$ the computations are similar in spirit, but easier.

\subsubsection{Linearisation of the mean curvature}

In this section we want to compute the discretised version of (3.1), that is the linear approximation of the curvature of a normal perturbation of a surface. For a general perturbation of a smooth curve such a formula can be found in [62]. 
Fix a time $t$ and use $V_{i}$ to denote the velocities of the vertices $z_{i}$. We seek coefficients $l_{i j}$ such that for the operator $L$ as defined in (3.1) we have

$$
L V\left(z_{i}, t\right) \approx \sum_{j} l_{i j} V_{j}
$$

Let tildes denote quantities referring to the configuration at the next time step. In particular, the vertices are given by $\tilde{z}_{i}=z_{i}+h V_{i} N_{i}$. Denote the mean curvature by $\kappa_{i}=\kappa\left(z_{i}\right)$ and by $\tilde{\kappa}_{i}=\kappa\left(\tilde{z}_{i}\right)$. The coefficients are then given by

$$
\left.\frac{d \tilde{\kappa}_{i}}{d h}\right|_{h=0}=\sum_{j} l_{i j} V_{j}
$$

and the coefficients $l_{i j}$ depend only on the vertices $z_{k}$.

Now assume $n=3$, for $n=2$ the following is much easier. Let tildes denote quantities referring to the configuration at the next time step. In particular, the vertices are given by $\tilde{z}_{i}=z_{i}+h V_{i} N_{i}$ and the mean curvature is given by $\tilde{\kappa}_{i}=\left(\nabla_{\tilde{z}_{i}} \tilde{A}_{i} \cdot \tilde{N}_{i}\right) /\left(\tilde{A}_{i} / 3\right)$. Also, $\nabla_{\tilde{z}_{i}} \tilde{A}_{i} \cdot \frac{d}{d h} \tilde{N}_{i}=0$ because $\tilde{N}_{i}$ is a unit vector and $\nabla_{\tilde{z}_{i}} \tilde{A}_{i}$ is parallel to $\tilde{N}_{i}$. Hence we define the $l_{i j}$ via

$$
\sum_{j} l_{i j} V_{j}=\left.\frac{d}{d h} \tilde{\kappa}_{i}\right|_{h=0}=\frac{\left.\frac{d}{d h}\left(\nabla_{\tilde{z}_{i}} \tilde{A}_{i}\right)\right|_{h=0} \cdot N_{i}}{A_{i} / 3}-\left.\frac{\nabla_{z_{i}} A_{i} \cdot N_{i}}{A_{i}^{2} / 3} \cdot \frac{d}{d h} \tilde{A}_{i}\right|_{h=0} .
$$

Standard computations lead to

$$
\begin{aligned}
& \left.\frac{d}{d h}\left(\nabla_{\tilde{z}_{i}} \tilde{A}_{i}\right)\right|_{h=0}= \\
& \quad \frac{1}{2} \sum_{\operatorname{star}\left(z_{i}\right)}\left\{-\frac{N_{k} \wedge\left(z_{j}-z_{i}\right)-N_{i j k}\left(N_{i j k} \cdot\left(N_{k} \wedge\left(z_{j}-z_{i}\right)\right)\right)}{\left|\left(z_{j}-z_{i}\right) \wedge\left(z_{k}-z_{i}\right)\right|} \wedge\left(z_{k}-z_{j}\right) V_{k}\right. \\
& \quad-\frac{N_{j} \wedge\left(z_{i}-z_{k}\right)-N_{i j k}\left(N_{i j k} \cdot\left(N_{j} \wedge\left(z_{i}-z_{k}\right)\right)\right)}{\left|\left(z_{j}-z_{i}\right) \wedge\left(z_{k}-z_{i}\right)\right|} \wedge\left(z_{k}-z_{j}\right) V_{j} \\
& \quad-\frac{N_{i} \wedge\left(z_{k}-z_{j}\right)-N_{i j k}\left(N_{i j k} \cdot\left(N_{i} \wedge\left(z_{k}-z_{j}\right)\right)\right)}{\left|\left(z_{j}-z_{i}\right) \wedge\left(z_{k}-z_{i}\right)\right|} \wedge\left(z_{k}-z_{j}\right) V_{i} \\
& \left.\quad+N_{i j k} \wedge N_{k} V_{k}-N_{i j k} \wedge N_{j} V_{j}\right\}
\end{aligned}
$$

and to

$$
\begin{gathered}
\left.\frac{d}{d h} \tilde{A}_{i}\right|_{h=0}=-\frac{1}{2} \sum_{\operatorname{star}\left(z_{i}\right)}\left\{N_{i j k} \cdot\left(N_{k} \wedge\left(z_{j}-z_{i}\right)\right) V_{j}+N_{i j k} \cdot\left(N_{j} \wedge\left(z_{i}-z_{k}\right)\right) V_{k}\right. \\
\left.+N_{i j k} \cdot\left(N_{i} \wedge\left(z_{k}-z_{j}\right)\right) V_{i}\right\} .
\end{gathered}
$$

The sums above are over all positively oriented facets $\left\{z_{i}, z_{j}, z_{k}\right\}$ of the surface contained in the star of $z_{i}$. A bit more notation makes it easier to write down specific formulae for the $l_{i j}$. We define

$$
\begin{aligned}
f_{i j k} & =N_{i j k} \cdot\left(N_{i} \wedge\left(z_{k}-z_{j}\right)\right), \\
F_{i j k} & =N_{i} \wedge\left(z_{k}-z_{j}\right)-f_{i j k} N_{i j k}, \\
A_{i j k} & =\left|\left(z_{j}-z_{i}\right) \wedge\left(z_{k}-z_{i}\right)\right|,
\end{aligned}
$$




$$
m_{i j k}=N_{i j k} \cdot\left(N_{j} \wedge N_{k}\right) .
$$

Then

$$
l_{i i}=\frac{1}{2 A_{i}} \sum_{\operatorname{star}\left(z_{i}\right)}\left(\frac{3}{A_{i j k}}\left|F_{i j k}\right|^{2}+\kappa_{i} f_{i j k}\right),
$$

and if $\left\{z_{i}, z_{l}, z_{j}\right\}$ and $\left\{z_{i}, z_{j}, z_{k}\right\}$ determine two adjacent positively oriented facets of $\operatorname{star}\left(z_{i}\right)$,

$$
\begin{aligned}
l_{i j}= & \frac{1}{2 A_{i}}\left(\frac{3}{A_{i j k}} F_{i j k} \cdot F_{j k i}+\frac{3}{A_{i l j}} F_{i l j} \cdot F_{j i l}\right. \\
& \left.+3\left(m_{j k i}+m_{j i l}\right)+\kappa_{i}\left(f_{j k i}+f_{j i l}\right)\right) .
\end{aligned}
$$

All other $l_{i j}$ are zero.

\subsection{Tangent disks}

Now that the manifold has been spatially discretised we indicate how to compute the $s_{i j}$ as defined in (3.7). For $n=3$ the surface patches $\Gamma_{i}$ will be approximated by planar disks $D_{i}$ perpendicular to $N_{i}$, having $z_{i}$ as their centre, and having area equal to a third of the area of $\operatorname{star}\left(z_{i}\right)$, that is the radii satisfy $\pi R_{i}^{2}=A_{i} / 3$. For $n=2$ one can use straight line segments with length equal to one-half of the sum of the distance to the two neighbouring vertices. Therefore we use

$$
s_{i j}=\frac{1}{\left|D_{i}\right|} \int_{D_{i}} \int_{D_{j}} k(x, y) d \sigma_{y} d \sigma_{x} .
$$

\section{Applicable models}

In this section we will describe three free boundary problems that fall within the characterisation as gradient flows. The notation is the same as in section 2.2.

\subsection{Averaged mean curvature flow}

This is the obvious example which arises by choosing $S$ to be the identity map.

$$
V=-(\kappa-\bar{\kappa}) \text {. }
$$

\subsection{Two-phase Mullins-Sekerka flow in $\mathbb{R}^{n}$}

This model arises when one chooses $S$ to be the single layer potential on the interface $\Gamma$. More precisely, let

$$
r(x)= \begin{cases}\frac{1}{2 \pi} \ln |x| & \text { for } n=2 \\ -\frac{1}{4 \pi}|x|^{-1} & \text { for } n=3\end{cases}
$$

and set

$$
S V(x)=\int_{\Gamma} r(x-y) V(y) d \sigma_{y}
$$


Potential theory allows one to infer many properties of this operator $S$, and of its inverse (see for example [41]). In particular $S V$ is a harmonic function off $\Gamma$, which is continuous across the interface and which has a jump in the normal derivative across $\Gamma$ equal to $V$. Using properties of $S V$ at infinity one can show that $S$ is invertible, and hence one determines that the gradient flow $V=-S^{-1}(\kappa-\bar{\kappa})$ will satisfy the following problem,

$$
\left\{\begin{aligned}
\Delta_{x} u(x, t) & =0 & & \text { in } \mathbb{R}^{n} \backslash \Gamma(t), \\
u(x, t)-\bar{\kappa}(t) & =O\left(1 /|x|^{2}\right) & & \text { as }|x| \rightarrow \infty, \\
u(x, t) & =\kappa(x, t) & & \text { on } \Gamma(t), \\
{\left[\partial_{N} u(x, t)\right] } & =V(x, t) & & \text { on } \Gamma(t) .
\end{aligned}\right.
$$

Here $u$ is an auxiliary function and $\left[\partial_{N} u\right]$ denotes the jump of the outer normal derivative of $u$ across $\Gamma$. The second line in the system assumes $n=3$, for $n=2$ it should be replaced by $|\nabla u(x, t)|=O\left(1 /|x|^{2}\right)$ as $|x| \rightarrow \infty$. If $\mathbb{R}^{n}$ is replaced by a bounded region, then the second line in the system needs to be replaced by a zero-Neumann boundary condition. Existence and regularity of smooth solutions on a bounded domain have recently been established by Escher and Simonett [31], and independently and at the same time by Chen, Hong, and Yi [21].

A physical interpretation of this model arises from spinodal decomposition where a chemical mixture is cooled into an unstable state. Separation into (two) different but miscible phases occurs by diffusion against the concentration gradients. The nonlinear Cahn-Hilliard equation models this process. It contains an interaction parameter, which one may think of as describing the width of the layer between the two regions containing mostly one phase or the other. It has been shown that the two-sided Mullins-Sekerka model arises as a singular limit when the interaction parameter approaches zero [3, 61].

\subsection{Two-phase Hele-Shaw flow in $\mathbb{R}^{n}$}

Take the operator $S^{-1}$ to be the double layer potential on $\Gamma$, that is for the function $r(x)$ from (4.2) define

$$
S^{-1} V(x)=\int_{\Gamma}<\nabla_{y} r(x-y), N(y)>V(y) d \sigma_{y},
$$

where $N$ denotes the outer unit normal. Using well-known properties of the double layer potential, $S^{-1} V$ is an harmonic function off $\Gamma$, which has a discontinuity across $\Gamma$ but also has a well-defined normal derivative on $\Gamma$, and that latter is equal to $-V$. In particular $S^{-1}$ is invertible, so that its notation as an inverse is justified. These facts leads to the following description of the arising gradient flow (as in the previous section $u$ is an auxiliary function),

$$
\left\{\begin{aligned}
\Delta_{x} u(x, t) & =0 & & \text { on } \mathbb{R}^{n} \backslash \Gamma(t), \\
u(x, t) & =O\left(1 /|x|^{2}\right) & & \text { as }|x| \rightarrow \infty, \\
{[u(x, t)] } & =-\kappa(x, t) & & \text { on } \Gamma(t), \\
{\left[\partial_{N} u(x, t)\right] } & =0 & & \text { on } \Gamma(t), \\
\partial_{N} u(x, t) & =V(x, t) & & \text { on } \Gamma(t) .
\end{aligned}\right.
$$

Physically this model describes the movement of the interface $\Gamma$ of two viscous incompressible immiscible fluids with all parameters set to unity; in particular the viscosities 
of the two fluids are assumed to be equal. Balancing forces at a point of the interface between the two fluids leads to $[u]=-\kappa$ where $-u$ represents the pressure of the liquids and $[u]$ stands for the jump of $u$ from the inside to the outside of $\Gamma$. The incompressibility of the fluids implies $\Delta u=0$. Continuity implies $\left[\partial_{N} u\right]=0$ at the interface. The fluids flow against the pressure gradient. Again, the second line of the system assumes $n=3$ and can be adjusted for the case $n=2$, or for a bounded domain, as described in the section above for the Mullins-Sekerka flow. The incompressibility and immiscibility of the fluids also implies that the volume enclosed by each component $\Gamma_{i}(t)$ of $\Gamma(t)$ is preserved, that is $\int_{\Gamma_{i}} V(x, t) d \sigma_{x}=0$ for all $i$. For this model it is therefore natural to work on a (dense) subspace of $\mathbf{L} \equiv \mathbf{L}_{2}^{0}\left(\Gamma_{1}\right) \times \mathbf{L}_{2}^{0}\left(\Gamma_{2}\right) \times \cdots \times \mathbf{L}_{2}^{0}\left(\Gamma_{m}\right) \subset \mathbf{L}_{2}^{0}(\Gamma)$ for a given surface $\Gamma$.

\section{Implementation: The averaged mean curvature flow}

This is the easiest example of volume preserving flows and is just meant as an illustration that the proposed numerical scheme is at all sensible. The two examples below have been chosen from the literature as they illustrate the formation of a singularity. Other applications of the scheme will appear elsewhere.
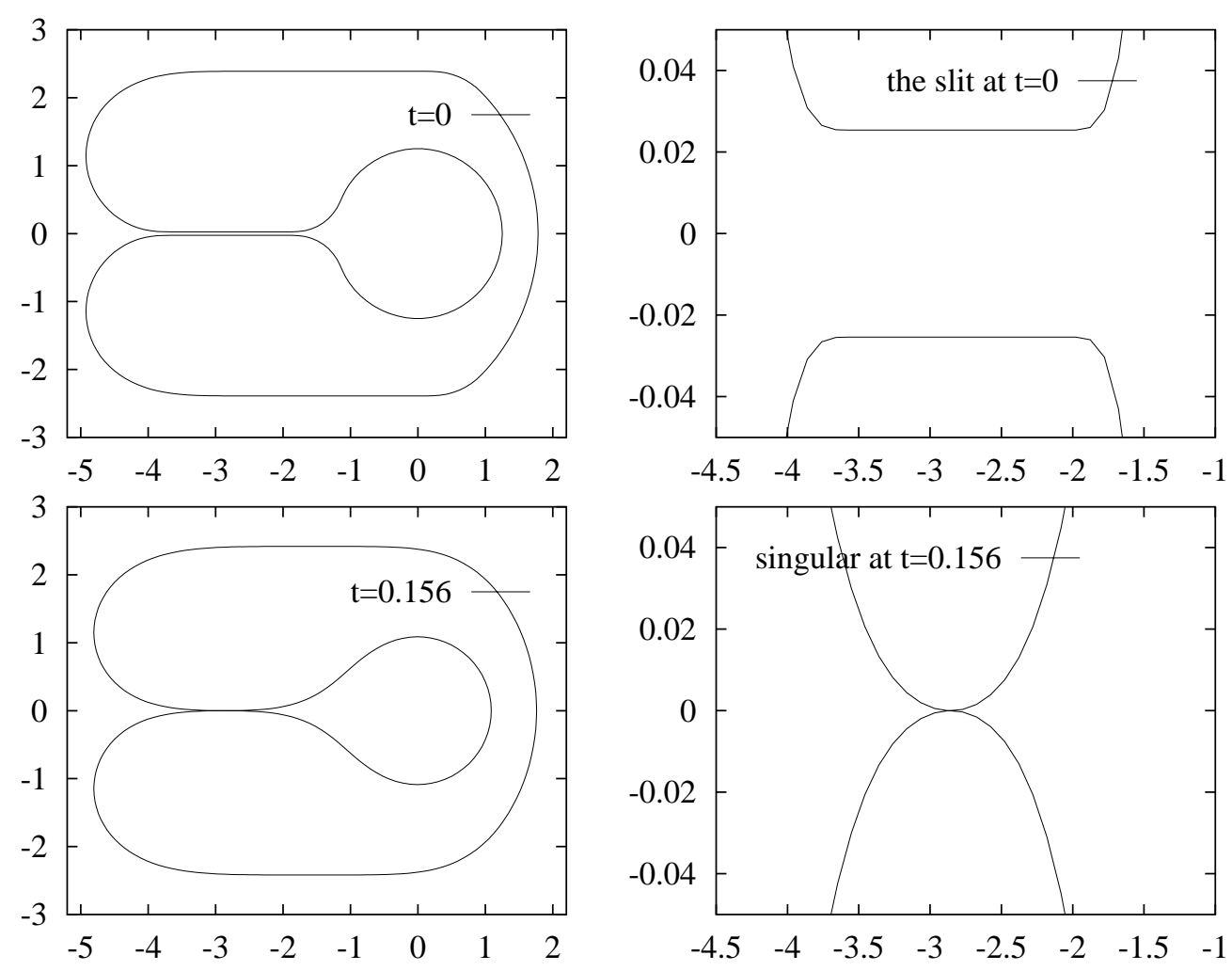

Figure 1 . At time $t=0.156$ the singularity has formed. 



Figure 2. Allowing the curve to become immersed.

\subsection{The example of Gage in two dimensions}

It is known that the (non-averaged) mean curvature flow preserves convexity [43, 52], and that plane curves will become convex [44] before they shrink to a point. However, for the averaged mean curvature flow the behaviour is different. The following example was first mentioned in the paper of M. Gage [42] for the problem

$$
V=-\left(\kappa-\frac{2 \pi}{L}\right) \text {. }
$$


Here of course $L$ is the length of the curve, and it is used that

$$
\int_{\Gamma} \kappa(s) d s=2 \pi
$$

for any simple closed positively oriented curve in the plane. Hence in our notation $\bar{\kappa}=$ $2 L / \pi$, so that this is exactly the flow from section 4.1 . The idea of the example is that on straight parts of a curve the curvature is zero, and hence because of the subtracted average one obtains an outward tendency. Choosing a figure with a narrow slit one should obtain a curve evolving to a self-intersection, see Figure 1.
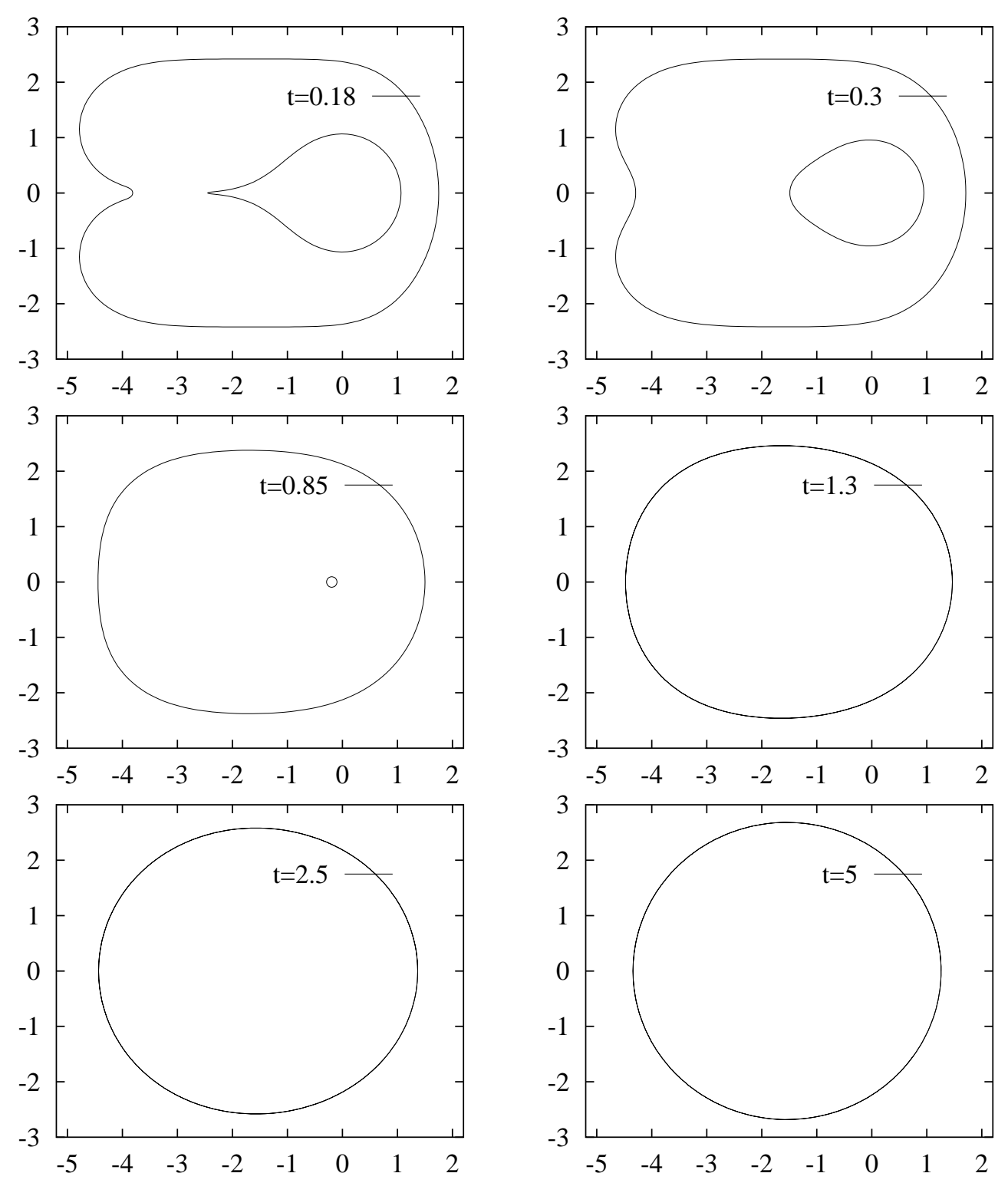

Figure 3. Allowing the curve to become disconnected. 


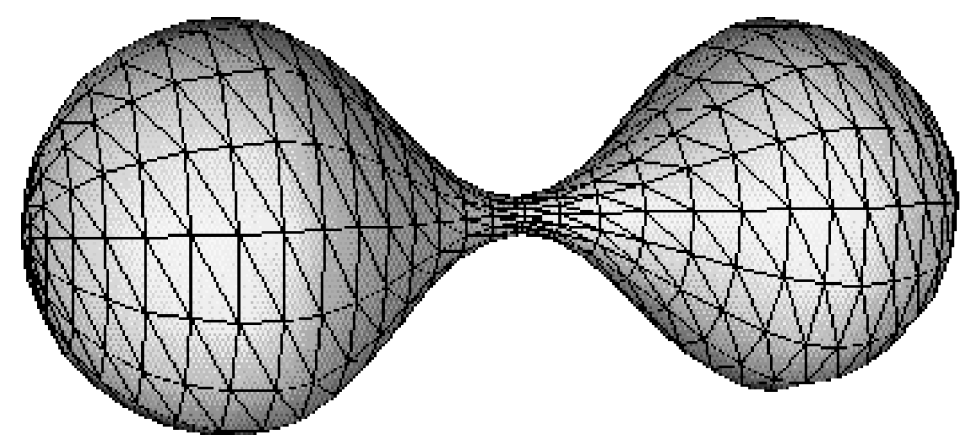

Figure 4. The initial surface with a thin neck. A cross section consists of a circular arc, one period of a cosine wave, and another circular arc. The length of the figure is about 10.68 units, the diameter of the neck is 2.2 units.

The evolution of the curve can be continued as an immersion due to the local character of the equation. As the evolution has the tendency to generate convex shapes (for which $\kappa$ is positive) the curves are expected to separate again, and to finally approach a circular limit shape, see Figure 2.

Another way of dealing with the singularity is to let the curve pop into two components, which leads to a configuration that is topologically an annulus, which will finally evolve back into a simply connected region, and then into a circle, see Figure 3.

\subsection{The example of Grayson in three dimensions}

In three space dimensions one may consider a dumbbell with a sufficiently thin neck. The mean curvature at the neck will be rather large, forcing a movement inwards, which should in due course create a singularity. This was analytically proved by Grayson [45] for the non-averaged mean curvature flow. Here is a numerical illustration of this example for the averaged mean curvature flow. Short term existence of classical solutions for the averaged mean curvature flow was established recently in [35], but there is no analytic result so far rigorously proving the development of the singularity. This example has also been numerically investigated by Blowey and Elliott [13], who use a phase-field approach. Their computations use the axial symmetry to reduce the computational complexity, while the numerical method described herein does not use any symmetry. Of course, Blowey and Elliott come to the same numerical conclusion, namely pinch-off at the neck. The graphics were generated using geomview, a graphics package produced at the Geometry Center at the University of Minnesota [59].

At this point the numerical simulation was stopped and restarted with two coinciding points located at the singularity, one connected to the left half and one connected to the right half of the figure, respectively. Then the simulation was allowed to continue.

Given any collection of spheres which evolve under the averaged mean curvature flow, one finds-provided the spheres are far enough from each other so that they do not coalesce - that the smaller spheres shrink, and the larger ones grow [64]. This has been termed - somewhat humorously - the survival of the fattest. In particular, the two approximate spheres above cannot be expected to be stable, if only because of numerical 
perturbation, see also similar remarks in [13]. For the case of two exact spheres it is straightforward to write down a system of ordinary differential equations for the two radii; the difference of the two differential equations can be used to obtain an upper bound for the time the smaller sphere will survive. For the experiment above, by chance, it is the left sphere that ultimately shrinks to a point.

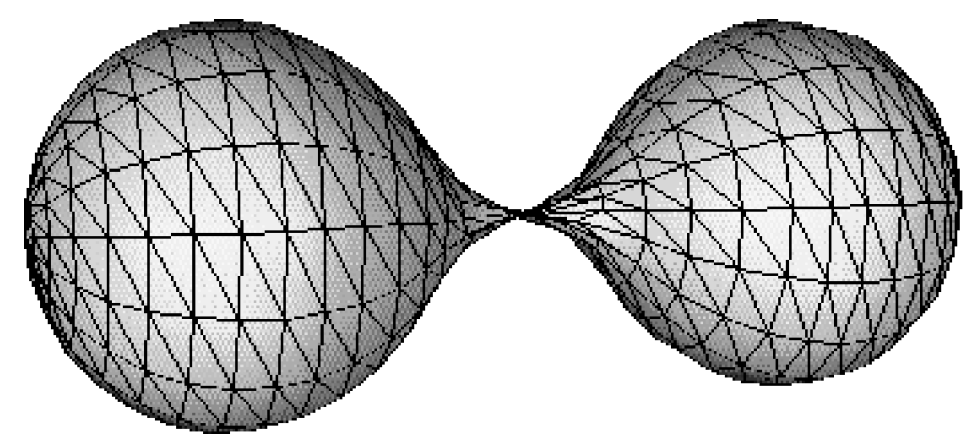

FiguRE 5. The thin neck has contracted to a point at about $t=0.65$.

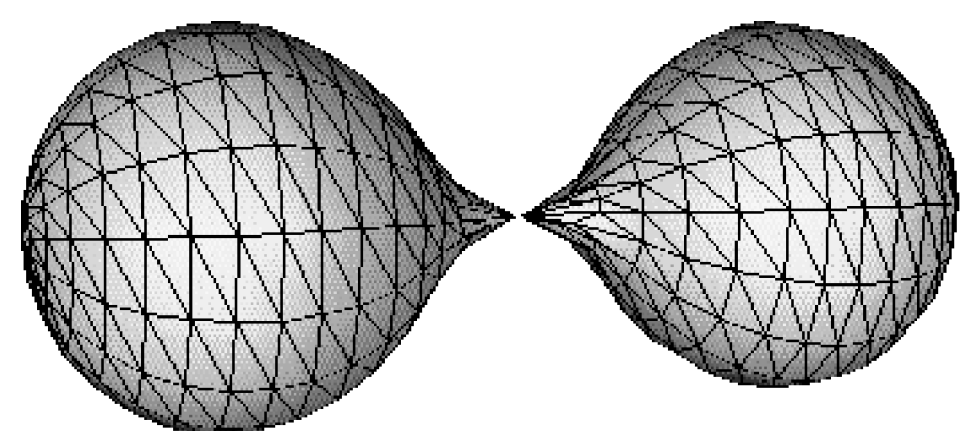

FIgURE 6 . The thin neck has popped apart at about $t=0.67$.
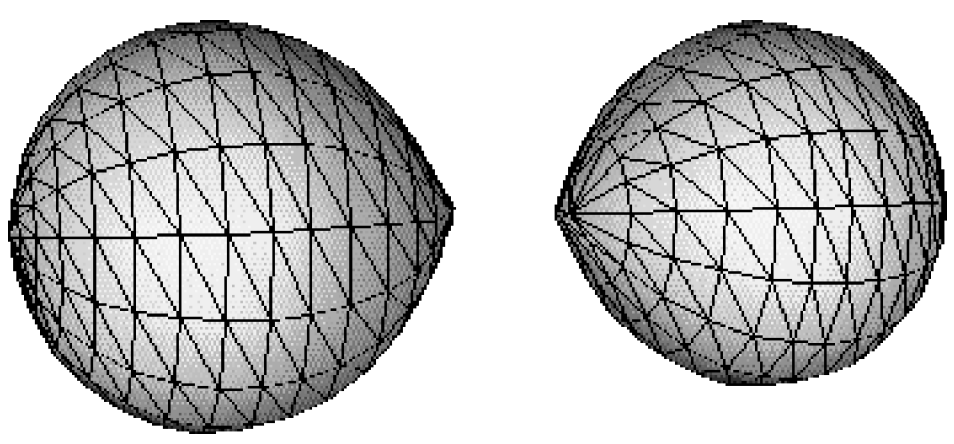

Figure 7. Evolving towards two spheres at $t=1.15$. 

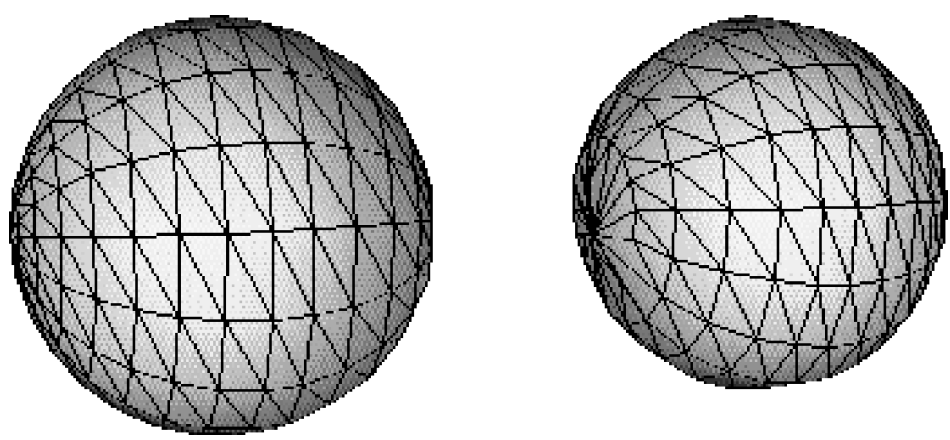

Figure 8. The configuration at $t=3.65$.

\section{Final remarks}

As the theory stands at this point, each step involves computing the inverse of a matrix. The number of floating point operations necessary to invert the (full) matrix is of cubic order in the number of vertices. However, for the example of the (averaged or not) mean curvature flow in two space dimensions one will get banded matrices due to the local structure of the problem (this assumes one numbers the vertices consecutively on the curve). Inversion of the resulting matrices is then only of linear order in the number of vertices. A similar result can be expected for axially symmetric configurations in three space dimensions. However, such a result cannot be easily achieved for arbitrary surfaces in three space dimensions, as there is no natural order of the vertices.

Also in two space dimensions, for the Mullins-Sekerka flow the matrix inversion problem can be avoided due to the special form of the kernel of the corresponding integral operator. The singular part of the kernel can be isolated and the inverse of it can be handled well using the Hilbert transform in combination with the fast Fourier transform [73]. Further research should show if something similar is also possible in higher space dimensions, or for the other problems. It is clear that inversion of a dense matrix at each step is unrealistic for large scale simulation. Of course, as the matrix, the right-hand side, and the solution of the linear system do not differ much from step to step, an iterative scheme should alleviate the computational cost somewhat. Better still would be an incorporation of a small scale decomposition technique, as developed by Hou, Lowengrup, and Shelly [50], or as used by Petersson [62]. Also, our numerical experiments have shown that the simulations are essentially not changing much with the time-step size, provided the time step is small enough to keep the experiment stable. Furthermore, this critical time-step size of course depends on the mesh size of the triangulation, the inner product, and on the size of the curvature and of its derivative. This is clearly an area for future research.

The proposed algorithm should allow to numerically determine geometric properties of the various flows, such as preservation of convexity, location of the centre of mass, pinch-off, or coalescence of separate components of the interface. It has been conjectured that the Mullins-Sekerka flow has similar behaviour as the mean curvature flow. However, contrary to this conjecture it has been shown in two dimensions that the Mullins-Sekerka flow does not preserve convexity $[54,56]$. We believe the same result is true in higher 
dimensions, but there is no proof. Also for the Mullins-Sekerka flow, there is the result that (not necessarily convex) initial configurations close to spheres (circles) converge exponentially fast to spheres (circles) [34] ([18]). In two dimensions there is numerical evidence that several particles could coalesce, and that a single particle could pinch off into two [10], but there are no results in three dimensions. Numerically it has been shown that the one-sided interior Hele-Shaw flow in two dimensions can drive a dumbbell shaped initial curve to self-intersection [6], and the characteristic fingering of the twosided Hele-shaw flow in the plane is a well-studied phenomenon, see the references in the introduction. However, there are no results for the behaviour of the two-sided Hele-Shaw flow in three dimensions that the author is aware of, and it is hoped that the proposed algorithm will alleviate this situation.

\section{Acknowledgements}

The author would like to express his gratitude towards the referees for their valuable suggestions, and in particular for pointing out the works of Petersson and of Blowey and Elliott.

\section{References}

[1] D. Adalsteinsson and J. A. Sethian, A fast level set method for propagating interfaces, J. Comput. Phys., 118 (1995), pp. 269-277.

[2] J. M. Aitchison And S. D. Howison, Computation of Hele-Shaw flows with free boundaries, J. Comput. Phys., 60 (1985), pp. 376-390.

[3] N. D. Alikakos, P. W. Bates, And X. Chen, The convergence of solutions of the CahnHilliard equation to the solution of the Hele-Shaw model, Arch. Rational Mech. Anal., 128 (1994), pp. 165-205.

[4] S. M. Allen and J. W. Cahn, A microscopic theory for antiphase boundary motion and its application to antiphase domain coarsening, Acta Metall., 27 (1970), pp. 1085-1095.

[5] F. Almgren, J. E. TAylor, And L. WAng, Curvature-driven flows: a variational approach, SIAM J. Control Optim., 31 (1993), pp. 387-438.

[6] R. Almgren, Singularity formation in Hele-Shaw bubbles, Phys. Fluids, 8 (1996), pp. 344352.

[7] R. Almgren, A. Bertozzi, And M. P. Brenner, Stable and unstable singularities in the unforced Hele-Shaw cell, Phys. Fluids, 8 (1996), pp. 1356-1370.

[8] R. Almgren, W.-S. Dai, And V. Hakim, Scaling behavior in anisotropic Hele-Shaw flow, Phys. Rev. Lett., 71 (1993), pp. 3461-3464.

[9] T. J. BARTh And J. A. Sethian, Numerical schemes for the Hamilton-Jacobi and level set equations on triangulated domains, J. Comput. Phys., 145 (1998), pp. 1-40.

[10] B. W. Bates, X. Chen, And X. Deng, A numerical scheme for the two phase MullinsSekerka problem, Electron. J. Differential Equations, 1995 (1995), pp. 1-27.

[11] P. W. Bates And P. C. Fife, The dynamics of nucleation for the Cahn-Hilliard equation, SIAM J. Appl. Math., 53 (1994), pp. 990-1008.

[12] J. T. Beale, T. Y. Hou, J. S. Lowengrub, And M. J. Shelley, Spatial and temporal stability issues for interfacial flows with surface tension. Theory and numerical methods for initial-boundary value problems, Math. Comput. Modelling, 20 (1994), pp. 1-27.

[13] J. F. Blowey AND C. M. ElliotT, Curvature dependent phase boundary motion and parabolic double obstacle problems, in Degenerate diffusions (Minneapolis, MN, 1991), Springer, New York, 1993, pp. 19-60. 
[14] - A phase-field model with a double obstacle potential, in Motion by mean curvature and related topics (Trento, 1992), de Gruyter, Berlin, 1994, pp. 1-22.

[15] K. E. Brakke, Surface Evolver, The Geometry Center, University of Minnesota, 1995.

[16] G. Caginalp, Stefan and Hele-Shaw type models as asymptotic limits of the phase-field equations, Phys. Rev. A, 39 (1989), pp. 5887-5896.

[17] J. W. Cahn and J. E. Hilliard, Free energy of a nonuniform system. III. Nucleation in a two-component incompressible fluid, J. Chem. Phys., 31 (1959), pp. 688-699.

[18] X. Chen, The Hele-Shaw problem and area-preserving curve-shortening motion, Arch. Rational Mech. Anal., 123 (1993), pp. 117-151.

[19] — Global asymptotic limit of solutions of the Cahn-Hilliard equation, J. Differential Geom., 44 (1996), pp. 262-311.

[20] X. Chen and C. M. Elliott, Asymptotics for a parabolic double obstacle problem, Proc. Roy. Soc. London Ser. A, 444 (1994), pp. 429-445.

[21] X. Chen, J. Hong, And F. Yi, Existence, uniqueness, and regularity of classical solutions of the Mullins-Sekerka problem, Comm. Partial Differential Equations, 22 (1996), pp. 17051727.

[22] Y.-G. Chen, Y. Giga, And S. Goto, Uniqueness and existence of viscosity solutions of generalized mean curvature flow equations, J. Differential Geom., 33 (1991), pp. 749-786.

[23] P. Constantin And M. Pugh, Global solutions for small data to the Hele-Shaw problem, Nonlinearity, 6 (1993), pp. 393-415.

[24] M. G. Crandall And T. M. Liggett, Generation of semigroups of nonlinear transformations on general Banach spaces, Amer. J. Math., 93 (1971), pp. 265-298.

[25] W.-S. DAi AND M. J. Shelley, A numerical study of the effect of surface tension and noise on an expanding Hele-Shaw bubble, Phys. Fluids, A 5 (1993), pp. 2131-2146.

[26] A. J. DeGregoria And L. W. Schwartz, A boundary-integral method for two-phase displacement in Hele-Shaw cells, J. Fluid Mech., 164 (1986), pp. 383-400.

[27] E. DiBenedetto And A. Friedman, The ill-posed Hele-Shaw model and the Stefan problem for supercooled water, Trans. Amer. Math. Soc., 282 (1984), pp. 183-204.

[28] J. Duchon And R. Robert, Évolution d'une interface par capillarité et diffusion de volume. I. Existence locale en temps, Ann. Inst. H. Poincaré Anal. Non Linéaire, 1 (1984), pp. 361-378.

[29] C. M. Elliott And V. Janovski, A variational inequality approach to Hele-Shaw flow with a moving boundary, Proc. Roy. Soc. Edinburgh, 88A (1981), pp. 93-107.

[30] C. M. Elliott, M. PaOlini, And R. Schätzle, Interface estimates for the fully anisotropic Allen-Cahn equation and anisotropic mean-curvature flow, Math. Models Methods Appl. Sci., 6 (1996), pp. 1103-1118.

[31] J. Escher And G. Simonett, On Hele-Shaw models with surface tension, Math. Res. Lett., 3 (1996), pp. 467-474.

[32] Classical solutions for Hele-Shaw models with surface tension, Adv. Differential Equations, 2 (1997), pp. 619-642.

[33] - Classical solutions of multidimensional Hele-Shaw models, SIAM J. Math. Anal., 28 (1997), pp. 1028-1047.

[34] - A center manifold analysis for the Mullins-Sekerka model, J. Differential Equations, 143 (1998), pp. 267-292.

[35] - The volume preserving mean curvature flow near spheres, Proc. Amer. Math. Soc., 126 (1998), pp. 2789-2796.

[36] L. C. Evans, H. M. Soner, And P. E. Souganidis, Phase transitions and generalized motion by mean curvature, Comm. Pure Appl. Math., 45 (1992), pp. 1097-1123.

[37] L. C. Evans And J. Spruck, Motion of level sets by mean curvature. I, J. Differential Geom., 33 (1991), pp. 635-681.

[38] A. Fasano And M. Primicerio, Blow-up and regularization for the Hele-Shaw problem, in Variational and Free Boundary Problems, A. Friedman and J. Spruck, eds., vol. 53 of IMA, Springer, New York, 1993, pp. 73-85. 
[39] P. C. Fife, Dynamical aspects of the Cahn-Hilliard equations, Barret Lectures, University of Tennessee, (1991).

[40] - Seminar notes on curve shortening, University of Utah, (1991).

[41] G. B. Folland, Introduction to differential equations, Princeton University Press, 1976.

[42] M. GaGe, On an area-preserving evolution equation for plane curves, Contemp. Math., 51 (1986), pp. 51-62.

[43] M. Gage and R. S. Hamilton, The heat equation shrinking convex plane curves, J. Differential Geom., 23 (1986), pp. 69-96.

[44] M. A. Grayson, The heat equation shrinks embedded plane curves to round points, J. Differential Geom., 26 (1987), pp. 285-314.

[45] — A short note on the evolution of a surface by its mean curvature, Duke Math. J., 58 (1989), pp. 555-558.

[46] M. E. GuRTin, Multiphase thermomechanics with interfacial structure, Arch. Rational Mech. Anal., 104 (1988), pp. 195-221.

[47] H. S. Hele-SHAw, The flow of water, Nature, 58 (1898), pp. 34-36.

[48] T. Y. Hou, Numerical solutions to free boundary problems, Acta numerica, (1995), pp. 335415.

[49] T. Y. Hou, Z. Li, S. Osher, AND H. ZhaO, A hybrid method for moving interface problems with application to the Hele-Shaw flow, J. Comput. Phys., 134 (1997), pp. 236-252.

[50] T. Y. Hou, J. S. Lowengrub, And M. J. Shelley, Removing the stiffness from interfacial flows with surface tension, J. Comput. Phys., 114 (1994), pp. 312-338.

[51] S. D. Howison, Complex variable methods in Hele-Shaw moving boundary problems, European J. Appl. Math., 3 (1992), pp. 209-224.

[52] G. Huisken, Flow by mean curvature of convex surfaces into spheres, J. Differential Geom., 20 (1984), pp. 237-266.

[53] _ - The volume preserving mean curvature flow, J. reine angew. Math., 382 (1987), pp. 35-48.

[54] U. F. MAYER, One-sided Mullins-Sekerka flow does not preserve convexity, Electron. J. Differential Equations, 1993 (1993), pp. 1-7.

[55] - Gradient flows on nonpositively curved metric spaces and harmonic maps, Comm. Anal. Geom., 6 (1998), pp. 199-253.

[56] — - Two-sided Mullins-Sekerka flow does not preserve convexity, in Mississippi State Conference on Differential Equations and Computational Simulations III, J. Graef, R. Shivaji, B. Soni, and J. Zhu, eds., Electronic J. Diff. Equ., 1998, pp. 171-180.

[57] U. F. MAYER And G. SimonetT, Self-intersections for the surface diffusion and the volume preserving mean curvature flow, Submitted, (1998).

[58] W. W. Mullins And R. F. SEKerkA, Morphological stability of a particle growing by diffusion and heat flow, J. Appl. Phys., 34 (1963), pp. 323-329.

[59] T. Munzner, S. Levy, And M. Phillips, Geomview, The Geometry Center, University of Minnesota, 1994.

[60] S. Osher AND J. A. Sethian, Front propagation with curvature-dependent speed: algorithms based on Hamilton-Jacobi formulations, J. Comput. Phys., 79 (1988), pp. 12-49.

[61] R. L. Pego, Front migration in the nonlinear Cahn-Hilliard equation, Proc. Roy. Soc. London Ser. A, 422 (1989), pp. 261-278.

[62] N. A. Petersson, Fast numerical computation of $2 D$ free surface jet flow with surface tension, J. Comp. Phys., 128 (1996), pp. 409-426.

[63] S. Richardson, Hele-Shaw flows with a free boundary produced by the injection of the fluid into a narrow channel, J. Fluid Mech., 56 (1972), pp. 609-618.

[64] J. Rubinstein And P. Sternberg, Nonlocal reaction-diffusion equations and nucleation, IMA J. Appl. Math., 48 (1992), pp. 249-264.

[65] L. W. Schwartz And A. J. DeGregoria, Two-phase flow in Hele-Shaw cells: numerical studies of sweep efficiency in a five-spot pattern, J. Austral. Math. Soc. Ser. B, 29 (1988), pp. $375-400$. 
[66] H. M. Soner, Convergence of the phase-field equations to the Mullins-Sekerka problem with kinetic undercooling, Arch. Rational Mech. Anal., 131 (1995), pp. 139-197.

[67] H. M. Soner ANd P. E. Souganidis, Singularities and uniqueness of cylindrically symmetric surfaces moving by mean curvature, Comm. Partial Differential Equations, 18 (1993), pp. 859-894.

[68] B. Sтотн, Convergence of the Cahn-Hilliard equation to the Mullins-Sekerka problem in spherical symmetry, J. Differential Equations, 125 (1996), pp. 154-183.

[69] J. E. TAYLOR, II-Mean curvature and weighted mean curvature, Acta metall. mater., 40 (1992), pp. 1475-1485.

[70] J. E. Taylor, J. W. Cahn, and C. A. Handwerker, I-Geometric models of crystal growth, Acta metall. mater., 40 (1992), pp. 1443-1474.

[71] P. W. VorheEes, Ostwald ripening of two-phase mixtures, Ann. Rev. Mater. Sci., 22 (1992), pp. $197-215$.

[72] P. W. Vorheees and M. E. Glicksman, Solution to the multi-particle diffusion problem with applications to Ostwald ripening, I and II, Acta Metall., 32 B (1984), pp. 2001-2030.

[73] J. Zhu, X. Chen, And T. Y. Hou, An efficient boundary integral method for the MullinsSekerka model, J. Comput. Phys., 127 (1996), pp. 246-267. 